viral infection or cirrhosis. Successful treatment of HB patients is achieved by combining surgery and chemotherapy. However, approximately one-quarter of the children do not survive the disease. HB prognostic factors rely on intrahepatic tumor extension, multifocality, vascular invasion, and distant metastases, whereas prognostic significance of histopathologic criteria remains debated. In a previous study by integrating molecular and genetic data, we described a poor prognostic HB subtype with $\beta$-catenin activation and stem cell features that evoked early phases of liver development (C. Armengol et al, Cancer Cell, 2008).

Aim: To study the protein expression of liver progenitor markers in $\mathrm{HB}$ and to associate them with the activation of $\mathrm{Wnt} / \beta$-catenin signalling pathway, tumour proliferation rate and clinical and pathological features.

Methods: EpCAM, AFP, KRT19, $\beta$-catenin and Ki67 protein expression were assessed by immunohistochemistry in a tissue microarray including $\mathrm{HB}(\mathrm{n}=48$ patients, median age: 2 years, sera AFP range: $150-4,700,000 \mathrm{ng} / \mathrm{ml}$, mean follow-up: 63 months), adjacent non tumour liver $(n=10)$ and fetal liver $(n=6)$ samples. The majority of patients had received chemotherapy according the SIOPEL 3 protocol.

Results: Overexpression of the liver progenitor markers EpCAM, AFP and KRT19 was observed in 48, 34 and $32 \%$ of $\mathrm{HBs}$, respectively. Moreover, $85 \%$ of cases showed an activation of the Wnt/ $\beta$-catenin signaling pathway with cytoplasmic and/or nuclear expression of $\beta$-catenin. Tumours with EpCAM over expression showed concomitant AFP over expression ( $p=0.011$ ), high tumour proliferation rate $(p=0.005)$ and nuclear accumulation of $\beta$-catenin $(p=0.013)$. At clinical level, EpCAM over expression was associated with reduced disease free survival $(\log$ rank $=0.022)$ and poor response to chemotherapy $(\mathrm{p}=0.05)$.

Conclusions: The over expression of the progenitor markers, EpCAM and AFP but not KRT19, is a poor prognostic factor in patients with HB. EpCAM, a $\beta$-catenin target gene and a mitogenic signal transducer, could be a good target for designing new therapeutic approaches for HB patients showing poor response to current treatments.

\section{4 \\ ROLE OF FLT3 AND ITS POTENTIAL INTERACTION PARTNER HSP27 IN HEPATOCELLULAR CARCINOMA}

M.M. Aydin ${ }^{1}$, N.S. Bayin ${ }^{2}$, E. Akhan ${ }^{1}$, A. Can ${ }^{3}$, K.C. Akcali ${ }^{1} .{ }^{1}$ Molecular Biology and Genetics, Bilkent University, Ankara, Turkey; ${ }^{2} N Y U$ Langone Medical Center, NYU Sackler Institute, New York City, NY, USA; ${ }^{3}$ Histology and Embryology, Ankara University, Ankara, Turkey E-mail: merveaydin@bilkent.edu.tr

Regeneration ability of liver is known to be associated with the activation of resident oval cells. Previously our group showed that oval cell marker, FLT3 changes its cellular localization in response to partial hepatectomy and suggested a role in liver regeneration. In addition, FLT3 was shown to play an important role in cellular proliferation and activation of PI3K and Ras. Hsp27 on the other hand, is a small chaperone that has roles in the inhibition of apoptosis and overexpressed in a wide range of cancers increasing their metastatic potential. Due to the role of FLT3 in liver regeneration and cellular proliferation, we aimed to analyze the effect of FLT3 in hepatocellular carcinoma (HCC) both in vitro and in vivo and its interaction with Hsp27.

We used a human HCC cell line, Snu398, which has high FLT3 expression both at mRNA and protein levels. We performed in vivo (xenografts on nude mice) and in vitro (wound healing and matrigel invasion assays) experiments to reveal the effect of FLT3 on tumorigenic and invasive potential of cells after treating with FLT3 inhibitor K-252a, and knocking down with shRNA. After performing immunoblotting and immunostaining for Hsp27, coimmunoprecipitation was done to show its interaction with FLT3.
Additionally, subcellular localizations of FLT3 and Hsp27 were determined by confocal microscopy.

Treatment of Snu398 cells with FLT3 inhibitor, K-252a changed the cytoplasmic localization of FLT3 and resulted in its accumulation mainly in nucleus and endoplasmic reticulum. Migration and tumor forming abilities of inhibitor treated cells were found to be highly diminished as demonstrated by wound healing and matrigel invasion assays. In addition, significant diminution in tumorigenicity and invasiveness was also obtained after stable knockdown of FLT3. Our data also revealed that Hsp27 might be a potential interaction partner for FLT3 evidenced by similar expression pattern as well as subcellular localization. Coimmunoprecipitation and confocal microscopy results confirmed this interaction between FLT3 and Hsp27.

We propose that FLT3 may function as a link between liver regeneration and HCC, where the FLT3 expressing cells may induce cancer progression through the participation of a metastasis inducing and anti-apoptotic chaperone Hsp27.

\section{5}

\section{GAMMA SMOOTH MUSCLE ACTIN: AN EPITHELIAL} MESENCHYMAL TRANSITION MARIKER IN HEPATOCELLULAR CARCINOMA

N. Benzoubir ${ }^{1,2}$, A. Dos Santos ${ }^{1,2}$, C. Guillaume ${ }^{3}$, D. Samuel ${ }^{1,2,4}$, C. Brechot ${ }^{1,2}$, M.-F. Bourgeade ${ }^{1,2}$, C. Guettier ${ }^{1,2,3} \cdot{ }^{1}$ Unit 785, INSERM, ${ }^{2}$ UMR-S785, Univ Paris-Sud, Villejuif, ${ }^{3}$ Service Anatomopathologie, AP-HP Hôpital de Bicetre, Le Kremlin-Bicêtre, ${ }^{4}$ Centre Hepato-Biliaire, AP-HP Hôpital Paul Brousse, Villejuif, France

E-mail: nassima.benzoubir@inserm.fr

During fibrogenesis and cancer progression, epithelial cells can undergo an epithelial-mesenchymal transition (EMT) defined as a complex transcriptional reprogramming triggered by different stimuli including TGF- $\beta$ and accompanied by dramatic morphological changes. In this process, tumor cells shed their epithelial differentiation characteristics and acquire mesenchymal features. EMT has been associated with invasiveness and poor tumor prognosis. More recently, EMT has been related to the emergence of cancer stem cells. In primary liver cancers, the role of EMT is still controversial.

During EMT, epithelial markers such as E-cadherin are strongly decreased in tumor cells whereas expression of mesenchymal markers such as vimentin or smooth muscle actin (SMA) is increased. Two SMAs have been described: the $\alpha$ SMA and the $\gamma$ SMA. Until now, only aSMA has been involved in EMT. Using hepatoma cell lines, we observed that TGF- $\beta$-induced EMT resulted in $\gamma \mathrm{SMA}$ polymerization whereas $\alpha$ SMA expression was barely detected. Moreover, overexpression of EMT transcription factors such as Snail and Twist was sufficient to induce $\gamma$ SMA polymerization, strongly suggesting that this actin isoform represents a marker for EMT in hepatoma cells.

Parallely, in situ immunohistochemical study for $\alpha$ SMA and $\gamma \mathrm{SMA}$ was performed on paraffin sections from surgical specimens of 60 hepatocellular carcinoma (HCC). In agreement with in vitro data, $\alpha$ SMA was consistently negative in all the samples whereas $\gamma$ SMA was expressed focally in about 50\% of HCC with a membranous and/or cytoplasmic staining. In normal hepatocytes $\gamma$ SMA expression was restricted to biliary canaliculi. Interestingly, $\gamma \mathrm{SMA}$ expression was constantly present in ductular reactions observed in cirrhosis and hepatitis with massive necrosis, suggesting that $\gamma$ SMA could represent a marker of progenitor liver cells.

Put together, these results suggest that $\gamma$ SMA could be a novel EMT marker in HCC; the prognostic relevance of this marker in HCC is under investigation.

This work is supported by INSERM, ANRS and INCa. 